\title{
Reshaping the Second-Order Polar Response of Hybrid Metal- Dielectric Nanodimers
}

\section{Journal Article}

\section{Author(s):}

Renaut, Claude (D); Lang, Lukas (D); Frizyuk, Kristina; Timofeeva, Maria; Komissarenko, Filipp E.; Mukhin, Ivan S.; Smirnova, Daria; Timpu, Flavia (D); Petrov, Mihail; Kivshar, Yuri; Grange, Rachel

\section{Publication date:}

2019-02-13

Permanent link:

https://doi.org/10.3929/ethz-b-000319709

\section{Rights / license:}

In Copyright - Non-Commercial Use Permitted

\section{Originally published in:}

Nano Letters 19(2), https://doi.org/10.1021/acs.nanolett.8b04089

\section{Funding acknowledgement:}

163916 - All-Dielectric and Hybrid Nanostructures for Biophotonic Applications (SNF)

150609 - Nonlinear Core-Shell Nanomaterials for Photonic Applications (SNF)

714837 - Second-Order Nano-Oxides for Enhanced Nonlinear Photonics (EC) 


\section{Reshaping the Second-Order Polar Response of Hybrid}

\section{Metal-Dielectric Nanodimers}

Claude Renaut ${ }^{\dagger}$, Lukas Lang ${ }^{\dagger}$, Kristina Frizyuk ${ }^{\S}$, Maria Timofeeva ${ }^{\dagger}$, Mihail Petrov ${ }^{\S}$, Filipp E.

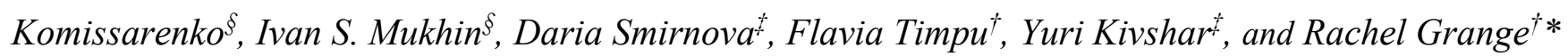

†Optical Nanomaterial Group, Institute for Quantum Electronics, ETH Zurich, 8093 Zurich, Switzerland

${ }^{\S}$ Department of Nanophotonics and Metamaterials, ITMO University, St. Petersburg 197101, Russia

$\$$ Nonlinear Physics Center, Australian National University, Canberra, ACT 2601, Australia

*E-mail: grange@phys.ethz.ch 
TABLE OF CONTENT GRAPHICS:

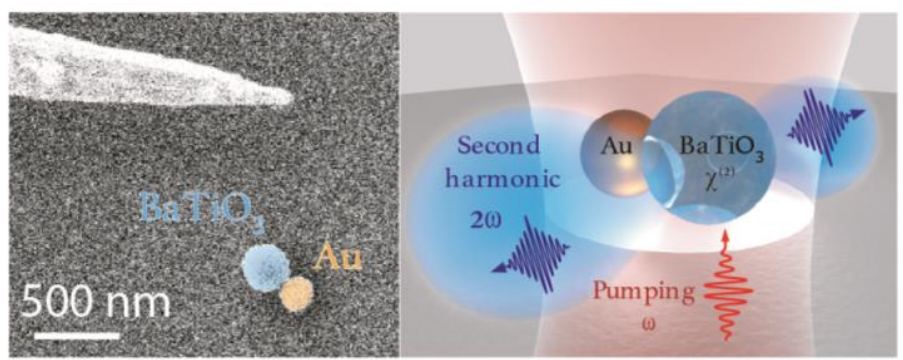




\section{ABSTRACT:}

We combine the field confinement of plasmonics with the flexibility of multiple Mie resonances by bottom-up assembly of hybrid metal-dielectric nanodimers. We investigate the electromagnetic coupling between nanoparticles in heterodimers consisting of gold and barium titanate $\left(\mathrm{BaTiO}_{3}\right.$ or $\left.\mathrm{BTO}\right)$ nanoparticles through nonlinear second-harmonic spectroscopy and polarimetry. The overlap of the localized surface plasmon resonant dipole mode of the gold nanoparticle with the dipole and higher-order Mie resonant modes in the BTO nanoparticle lead to the formation of hybridized modes in the visible spectral range. We employ the pick-and-place technique to construct the hybrid nanodimers with controlled diameters by positioning the nanoparticles of different types next to each other under a scanning electron microscope. Through linear scattering spectroscopy, we observe the formation of hybrid modes in the nanodimers. We show that the modes can be directly accessed by measuring the dependence of the second-harmonic generation (SHG) signal on polarization and wavelength of the pump. We reveal both experimentally and theoretically that the hybridization of plasmonic and Mieresonant modes leads to a strong reshaping of the SHG polarization dependence in the nanodimers, which depend on the pump wavelength. We compare the SHG signal of each hybrid nanodimer with the SHG signal of single BTO nanoparticles to estimate the enhancement factor due to the resonant mode coupling within the nanodimers. We report up to two orders of magnitude for the SHG signal enhancement compared to isolated BTO nanoparticles.

KEYWORDS: Second-harmonic generation, Hybrid nanoantennas, Polar-dependence, Plasmonics, Dielectrics, Barium Titanate 
Controlling the emission properties of optical antennas is achievable using different strategies but they remain strongly bound to plasmonics, even for nonlinear signal generation. ${ }^{1-4}$ Nonlinear generation in photonic nanostructures constantly attracts the interest of researchers seeking to enhance the efficiency of higher order effects and to control the emission of the generated light ${ }^{5}$. Recently, the focus in nonlinear generation switched from fully metallic nanostructures ${ }^{6-9}$ to dielectric and semiconductor resonant systems due to rapidly developing all-dielectric Mie-resonant nanophotonics. ${ }^{10,11}$ The intrinsic advantage of non-plasmonic resonant systems ${ }^{12-14}$ is the low losses and the enhanced second-order nonlinear response due to a bulk nonlinearity of materials. ${ }^{15-19}$ The enhancement of second-harmonic generation (SHG) in non-plasmonic systems possessing Mie resonances has been reported in semiconductors ${ }^{15,20}$ and lead-halide materials, which may potentially demonstrate up to $10^{-2}$ conversion efficiency of SHG. ${ }^{21}$ The progress in all-dielectric nanophotonics has stimulated the studies of hybrid structured systems ${ }^{22,23}$ formed by a metallic and dielectric parts, which combine the high field localization of plasmonics ${ }^{24}$ with the optical properties of dielectric systems. ${ }^{25}$ The metallic components have already demonstrated the ability to enhance nonlinear signals from dielectric nanoparticles in various hybrid systems. ${ }^{26-28}$ However, the interest in hybrid structures is not only supported by the ability to enhance the electromagnetic field but also to hybridize resonances formed by localized surface plasmon resonance, ${ }^{29}$ and volume Mie modes of dielectric nanoparticles. ${ }^{25,30,31}$ By controlling the interaction of metallic and dielectric nanoparticles, an additional level of tuning can be reached allowing for control over the elastic light scattering, ${ }^{32,33}$ which is also related to bi-anisotropy effect in nanodimers. ${ }^{34}$ In this work, we consider hybrid nanodimers consisting of a plasmonic nanoparticle and a dielectric resonant nanoparticle with non-centrosymmetric crystalline structure. The spherical geometry of the chosen nanoparticles allows to use Mie theory for modeling the optical response of individual nanoparticles. Note that we already showed the small influence of a non spherical shape on the scattering in oblate barium titanate $\left(\mathrm{BaTiO}_{3}\right.$ or BTO) nanoparticles. ${ }^{35}$ With experimental measurements of the linear scattering of the nanoparticles and nanodimers, we show the hybridization of the resonant stated. Finally, we demonstrate that the resonant hybrid states of the nanodimers can be efficiently probed through wavelength-dependent and polarization-dependent SHG measurements. The experiments and the modeling reveal the resonant 
contributions involved in the hybridization of the modes. The results can generate new applications in the engineering of the near-field control of the light through a far-field parameter, namely, the polarization of the laser source in the case of hybrid nanodimers. Moreover, the overlap of the resonances occurs in the visible range and leads to strong enhancements of the nonlinear signal.

Single BTO nanoparticles exhibit several Mie resonances in the visible range due to a refractive index above $2 .{ }^{35}$ The wavelength dependent scattering cross section of a $240 \mathrm{~nm}$ diameter single BTO nanoparticle under a plane wave illumination is shown in Figure 1a accompanied by the decomposition into multipoles given by Mie theory. The leading contributions to the scattering come from the magnetic dipole (MD) and magnetic quadrupole (MQ), with the electric dipole (ED) being very broad and embedded in the magnetic resonances. The modeled $200 \mathrm{~nm}$ diameter Au nanoparticle has a predominant localized surface plasmon resonance centered at $650 \mathrm{~nm}$, while the quadrupolar resonances are negligible in the studied range as shown in Figure 1b. We choose the sizes of the individual nanoparticles to overlap the positions of the dipole resonances: ED mode for gold and MD mode for BTO, and their near-field to match the amplitudes of their induced dipole moments. The experimental measurements of the scattering spectra of the individual nanoparticles with a home-built linear dark-field optical spectroscopy setup agree with the theoretical results of Figure 1a-b (see Methods). The experimental forward scattering curves of the individual nanoparticles are plotted with purple dots for a $210 \mathrm{~nm}$ diameter Au nanoparticle and red dots for the $280 \mathrm{~nm}$ diameter BTO nanoparticle in Figure 1c. Scanning electron micrographs of the optically measured nanoparticles are in insets of Fig. 1 (c). The normalized backward linear scattering curves of the individual nanoparticles are available in the supporting information. The typical features of the linear Mie scattering model of the single BTO are present on the measurements of the linear scattering shown with red dots in Figure 1c. The wide peak centered around $620 \mathrm{~nm}$ on the forward scattering belongs to the MD resonance. Another sharp peak is visible around $460 \mathrm{~nm}$ on the measured linear scattering curve and belongs to the MQ resonance. The measured forward scattering spectrum shows a resonance peak which resembles the MQ resonance of the modeling in Figure 1a. If we compare the modeled linear contributions of the Au nanoparticle with the measurements, we notice a resonance centered at $620 \mathrm{~nm}$ on the forward linear scattering spectrum, purple dots, Figure 1c. We also observe a 
second resonant contribution on the forward scattering of Au visible at $460 \mathrm{~nm}$, which corresponds to the forward scattering of the EQ mode of the model as shown in Figure 1b. The multipole decomposition obtained with Mie theory for the Au nanoparticle shows that the contribution of the EQ modes stays negligible in comparison to the ED mode Figure $1 \mathrm{~b}$. We highlight by a red shade the spectral range from 560 to $700 \mathrm{~nm}$ on the linear spectra of Figure 1 where we expect the hybridized mode to occur.
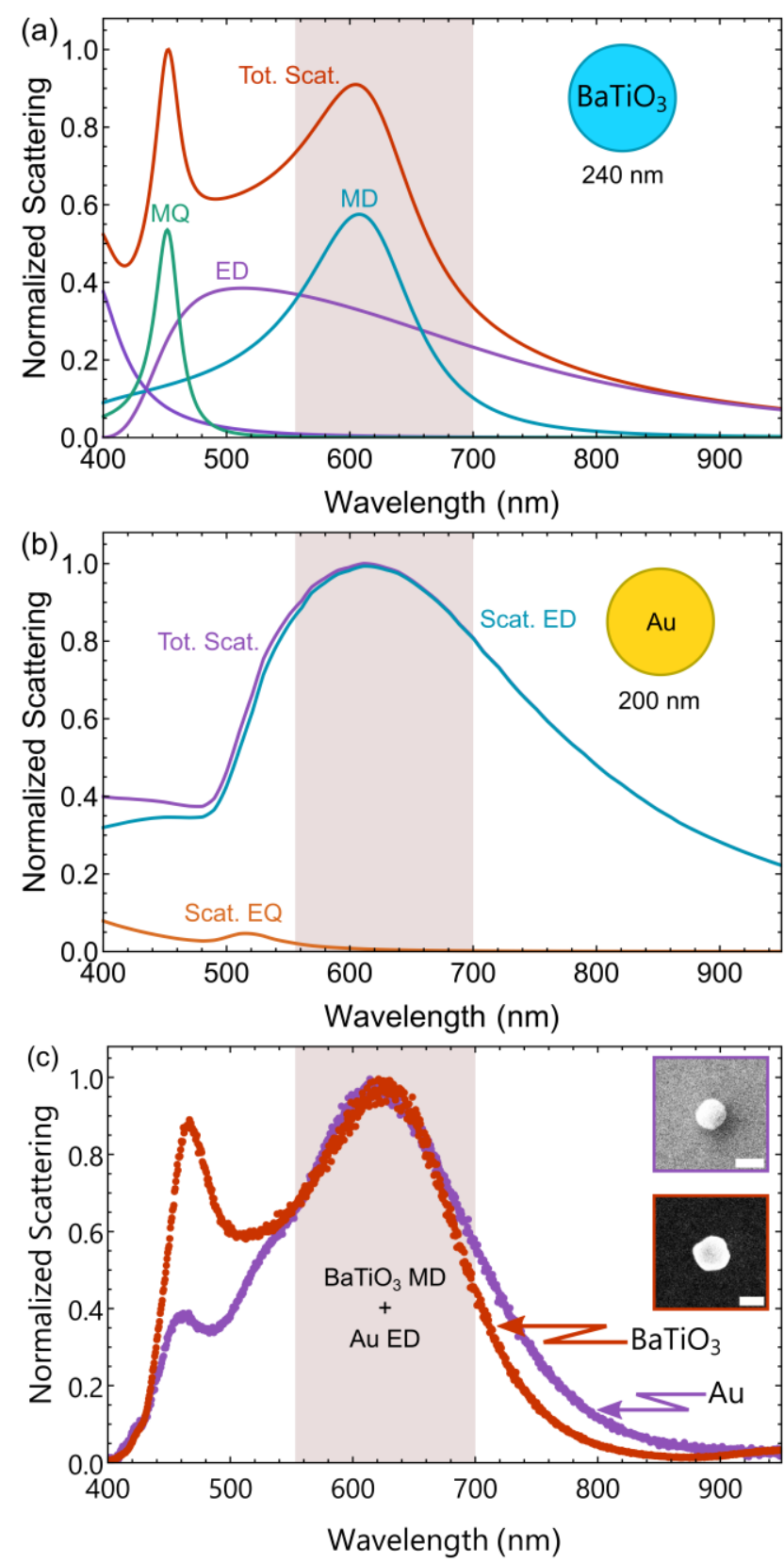

Figure 1. (a) Modeled scattering of a $240 \mathrm{~nm}$ diameter BTO nanoparticle under plane wave illumination with the decomposition into multipoles given by Mie theory. (b) Modeled scattering and absorption of a $200 \mathrm{~nm}$ diameter Au nanoparticle under plane wave illumination with the decomposition into multipoles given by Mie theory. (c) Experimentally measured forward linear scattering of a single $210 \mathrm{~nm}$ size $\mathrm{Au}$ (purple dots) and a single $280 \mathrm{~nm}$ size BTO (red dots) nanoparticles. The highlighted region of the spectra is showing the spectral range of the hybridized mode. In insets: SEM images of those single nanoparticles, the Au displayed in purple and the BTO in red, scale bars $200 \mathrm{~nm}$. 
The modeled scattering spectrum of the hybrid nanodimer is considering the experimental parameters of excitations and collections of the home-built dark-field spectroscopy setup and is shown with a solid light blue line in Figure 2a. In the inset of Figure 2a, we give a schematic view of the modeled nanodimer that matches with the sample sizes. The calculations are performed using the finite-elementmethod solver in COMSOL Multiphysics, which accounts for the material dispersion in Au and BTO. The hybridized mode constructed from the MD mode of the dielectric nanoparticle and ED mode of the plasmonic nanoparticle gives a response centered slightly below $700 \mathrm{~nm}$ in our model for a $280 \mathrm{~nm}$ BTO and a $212 \mathrm{~nm}$ Au nanoparticle Figure 2a.

The hybrid nanodimers are fabricated by the pick-and-place technique from Au and BTO nanoparticles. ${ }^{36,37}$ The single nanoparticles are electrostatically manipulated under the beam of an electron microscope with a sharp metallic tip and assembled on an indium tin oxyde covered glass substrate into hybrid nanodimers (supporting information). We have fabricated several nanodimers with varying sizes of BTO nanoparticles: D1 $(314 \mathrm{~nm})$, D2 $(298 \mathrm{~nm})$ and D3 $(330 \mathrm{~nm})$ as shown in the insets of Figure 2 (and with close-up SEM images in the supporting information Fig S10). The modeled linear scattering spectrum of the hybrid nanodimer (Figure 2a) is shown alongside the forward linear scattering measurements of the fabricated hybrid nanodimers D1, D2 and D3 in Figure 2b-d (backward scattering spectra can be found in the supporting information). 


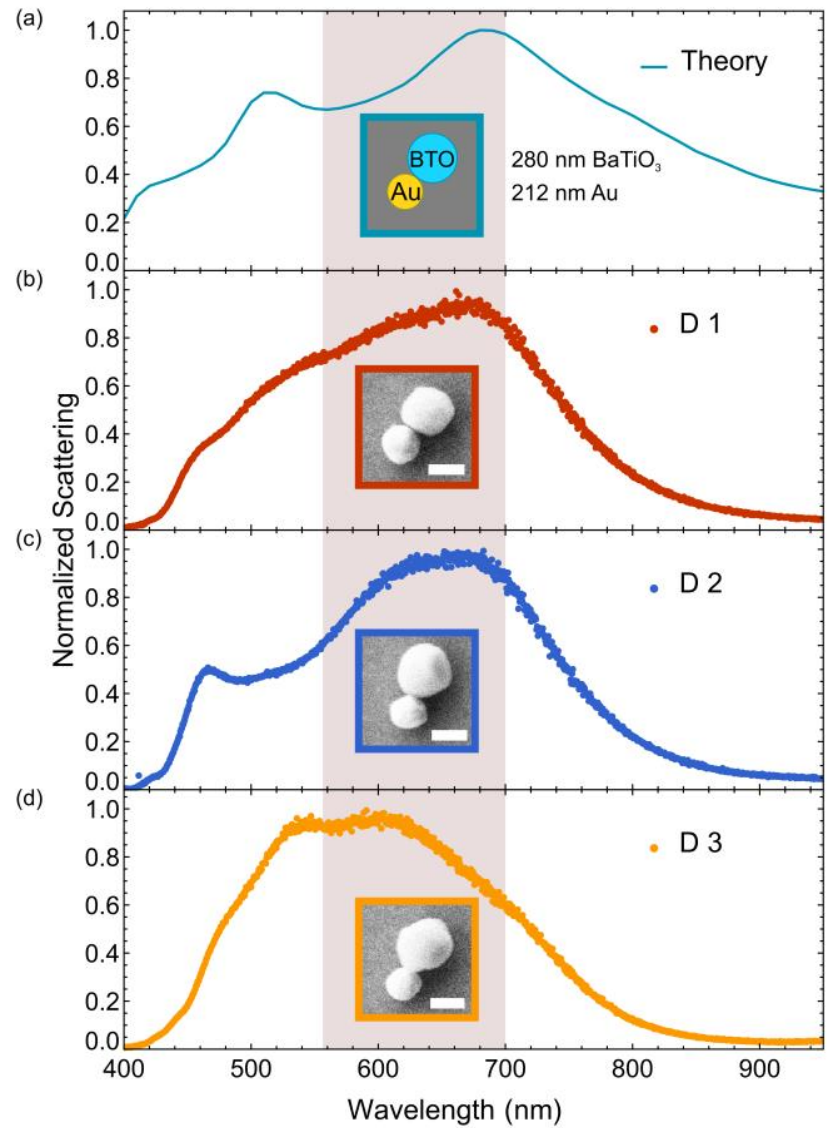

Figure 2. (a) Modeled forward (normalized) linear scattering of a nanodimer with a $\mathrm{Au}(212 \mathrm{~nm})$ and a BTO $(280 \mathrm{~nm})$ nanoparticles. Inset: a schematic view of the nanodimer and their corresponding materials and sizes. (b-d) Measured forward (normalized) linear scattering of three nanodimers D1 (red, b), D2 (blue, c) and D3 (orange, d) with sizes of Au/BTO particles of $213 \mathrm{~nm} / 314 \mathrm{~nm}, 200 \mathrm{~nm} / 298 \mathrm{~nm}$ and $200 \mathrm{~nm} / 330 \mathrm{~nm}$. In the insets: electron micrographs of each measured nanodimers. Scale bars are $200 \mathrm{~nm}$.

The trend of the measured forward linear scattering spectra of the nanodimers is corresponding to the modeled nanodimer scattering spectra. In the case of D1 and D2 we can differentiate two peaks. A higher intensity one at longer wavelength and a lower intensity one at shorter wavelength, they correspond to the modeled spectrum Figure 2a. The measured scattering spectrum of D3 shows less correspondence with the calculation than the first two nanodimers D1 and D2. The difference in peak positions of the measured spectra are due to uncertainties of measured sizes and distances between the nanoparticles of the nanodimers by electron microscopy used for the calculations. ${ }^{38,39}$ Based on the linear model for the single nanoparticles and the nanodimer, we conclude that the second peak slightly below $700 \mathrm{~nm}$ (Figure 2) coincides with the hybridized MD mode of BTO and the ED plasmonic mode of Au (supporting information). The linear scattering spectra reflect the mode hybridization within the nanodimers. 
To probe the hybridized mode of the nanodimers we use the polarimetry of the SHG signal generated by the hybrid nanodimers and we compare it with the polarimetry of the second-harmonic signal of single BTO nanoparticles with the same size as in the hybrid nanodimers. ${ }^{40}$ The polarimetry of the SHG signal gives a simple and sensitive way to identify the mode coupling. We know from the linear calculations and measurements that the nanodimers possess resonance features in the visible and in the near-infrared part of the spectrum (Figure 2). We perform second-harmonic characterization in transmission on a home-built setup, a detailed schematic of the experiment is presented in supporting information. The sample is positioned with the indium tin oxide (ITO) substrate perpendicularly to the pump laser propagation axis. We excite the sample with the laser through a lens from the back side of the ITO covered glass substrate. On the nanodimer side we collect the SHG signal with a microscope objective. We then image the SHG light onto a scientific CMOS silicon camera. The pump laser light is filtered out with bandpass or short-pass filters to detect solely the SHG signal from the nanodimer on the camera. We control the orientation of the linearly polarized fundamental laser light with the combination of a Glan-Taylor polarizer and a half-wave plate mounted on a rotation stage before the focusing lens. The optical power level is controlled via another half-wave plate before the linear polarizer. The SHG signals are recorded for every nanodimer with a $10 \mathrm{~nm}$ step on the $800-1400 \mathrm{~nm}$ excitation wavelength range separated in two ranges: (i) a Ti: Sapphire laser (laser) covers 800 to $1080 \mathrm{~nm}$ (ii) an optical parametric oscillator (OPO) covers 1040 to $1400 \mathrm{~nm}$ with a small overlap between 1040 and $1080 \mathrm{~nm}$. We coupled both pump beams in the same entry port of the optical setup. We reconstruct the SHG spectra from the measured far-field SHG images of the hybrid nanodimers. Every $50 \mathrm{~nm}$ (starting at $800 \mathrm{~nm}$ ), we record the polarization dependency of the SHG signal of the nanodimers. All the SHG data are normalized by the experimental parameters, the details of the optical setup and normalization procedure are available in supporting information.

The second-harmonic spectrum of the single $280 \mathrm{~nm}$ BTO nanoparticle presents in Figure 3a both measurement and calculations. We can see that for fundamental wavelength below $1050 \mathrm{~nm}$ a steep growth of SHG signal is present. The increase of the SHG signal originates from approaching the MD resonance mode of BTO at the fundamental wavelength. ${ }^{41}$ After $1050 \mathrm{~nm}$ a peak corresponding to the 
MQ resonance mode of the dielectric nanoparticle appears, the MD resonance of the dielectric nanoparticle remains low on the spectrum due to the symmetry of modes and the crystalline structure of BTO. We already highlight the spectral range from $1100 \mathrm{~nm}$ to $1400 \mathrm{~nm}$, which corresponds to the longwavelength hybridization region for nanodimers (Figure 3a). The measured polar dependence of the BTO nanoparticle has a typical four-lobe structure due to its crystal orientation on the whole wavelength range of the polar plot, (Figure 3b). ${ }^{42,43}$ The polar dependence of the SHG strongly depends on the orientation the crystalline lattice and particular resonant modes within the nanoparticle. ${ }^{44}$ As the particular orientation of the crystalline axis in the nanoparticle is unknown, we have performed numerical simulations of SHG polar dependence, identifying the exact orientation of the crystalline lattice. 


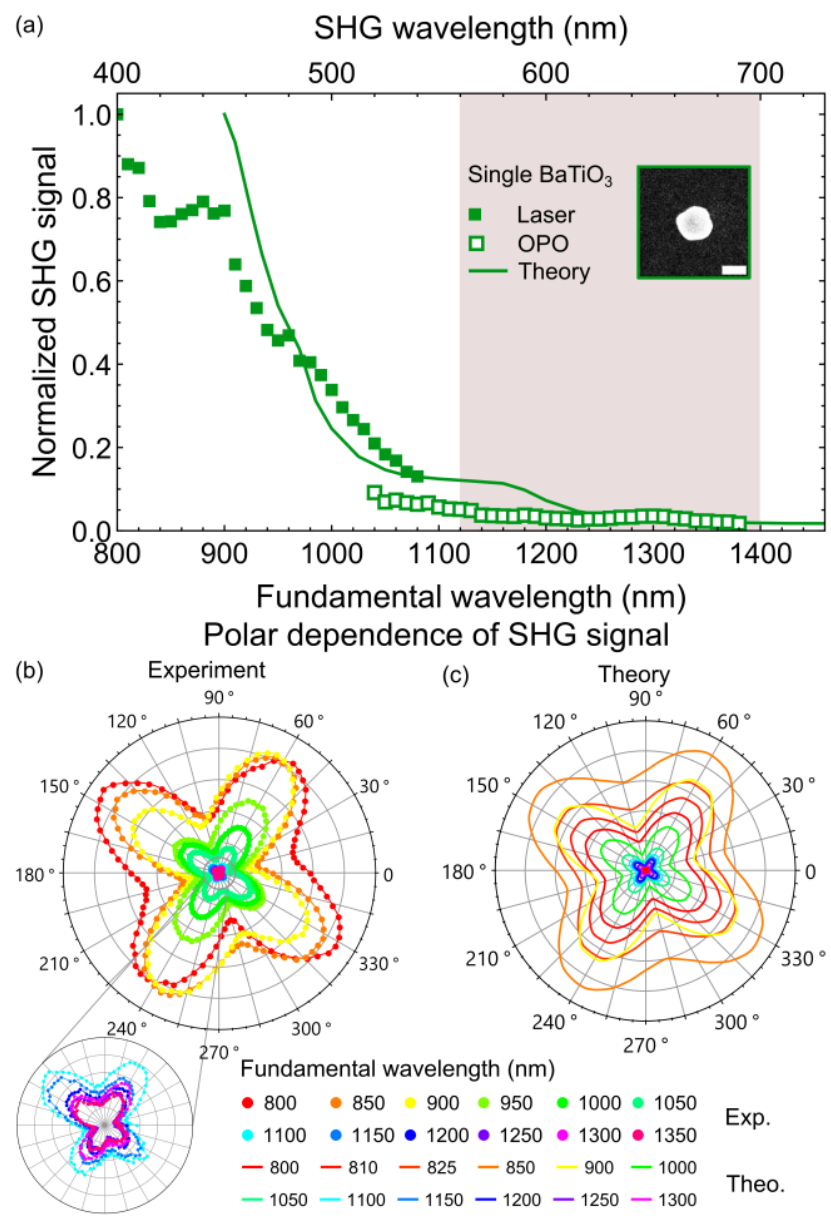

Figure 3. (a) Normalized SHG signal of a single BTO as a function of the fundamental wavelength. The solid green line shows the modeled spectrum and the green squares are the measurements points (filled laser, empty OPO). Electron micrograph of the particle in inset, scale bar $200 \mathrm{~nm}$. (b) Measured, (c) modeled polar dependence of the normalized SHG signal from a single BTO. The colors of the polar plots correspond to excitation wavelengths.

We then measure the second-harmonic spectrum of the nanodimer D1. As for the single BTO particle, the SHG spectrum of D1 is measured separately in two regions, the laser $(800-1080 \mathrm{~nm})$ and the OPO $(1040-1400 \mathrm{~nm})$. In the laser range, filled red dots, we observe a wide peak coming from the overlap of the ED modes from the plasmonic and the dielectric nanoparticles, Figure 4a. The same effect is visible on the numerical calculation, solid red line of Fig. 4 (a). The OPO range, empty red dots, has a more complicated response, a peak and valley then a plateau. The measured second-harmonic spectrum generally agrees with the two peaks of the modeled SHG spectrum (Figure 4a). In the OPO range, the angular dependence shows well pronounced two lobes shape oriented along the nanodimer long axis $110^{\circ}$ (Figure 4b). The orientation of the polar plot is due to the field enhancement along the long axis of the nanodimer which arises from the hot-spot close to the plasmonic nanoparticle and is visible for all measured nanodimers. We see in Figure 4b-c that the SHG polar dependences are rotated and reshaped 
due to the resonance of the hybridized mode. The shape of the polar diagram depends on the crystalline orientation of the BTO nanoparticle, which cannot be chosen during the pick-and-place fabrication, combined with the field enhancement encountered at the resonances of the nanodimers. We see that the measured data are supported by the results of numerical modeling in Figure $4 b-c$.

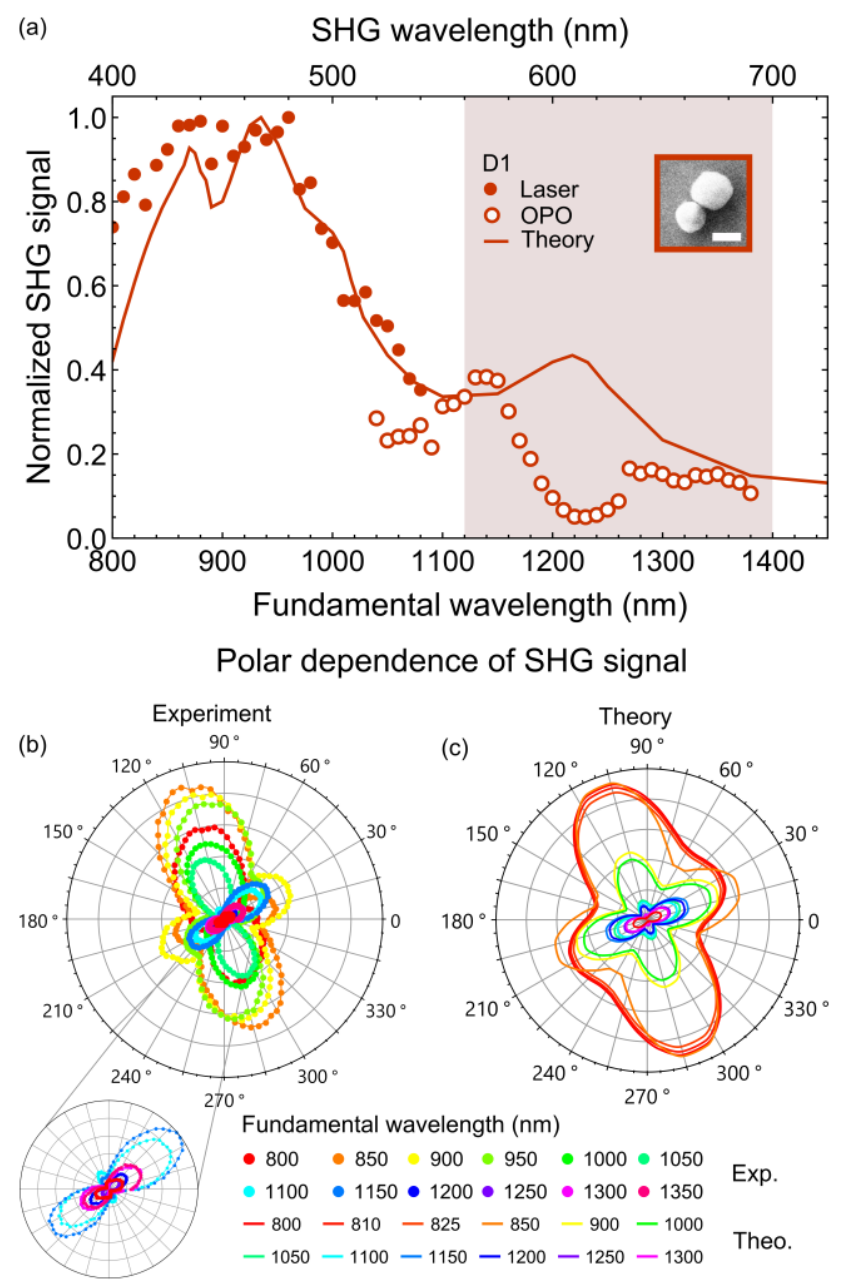

Figure 4. (a) Normalized SHG signal of nanodimer D1 as a function of the fundamental wavelength. The solid red line shows the modeled spectrum and the red dots are the measurements points (filled laser, empty OPO). Electron micrograph of D1 in inset, scale bar $200 \mathrm{~nm}$. (b) Measured, (c) modeled polar dependence of the normalized SHG signal from nanodimer D1. The colors of the polar plots correspond to excitation wavelengths.

Following the same methodology, we characterize the SHG response of nanodimer D2. The measured spectrum presents two peaks of SHG, one in the laser range (filled blue triangles) and the other in the OPO range (empty blue triangles) Figure 5a. Besides differences originating from the sizes and the spherical shape of the nanoparticles, the measured SHG spectrum corresponds to the theoretical model (blue line, Figure 5a). Indeed, in D2, the BTO nanoparticle is the biggest (300 nm diameter) and other modes can play a role in the SHG response. The polar dependent SHG data of D2, measurements in 
Figure $5 \mathrm{~b}$ and calculations in Figure 5c present a two lobes dependence with a $105^{\circ}$ rotation between the laser and the OPO pumping range. The change in the polar dependence is clear especially if we look at the $1000 \mathrm{~nm}$ polar plot and compare it to the $1100 \mathrm{~nm}$. At $1000 \mathrm{~nm}$ we observe a dipole like shaped polar plot which is different from the one at $1100 \mathrm{~nm}$, measured and modeled, Figure 5b-c. The sudden change in the polar dependence comes from the resonating behavior of the hybridized mode of the nanodimer combined with a specific orientation of the second order susceptibility tensor. We modeled the polar plots of the different nanodimers considering that the double resonance effect of the hybridized mode combines with the second order susceptibility tensor orientation. However, the measurements of the SHG far-field response give distinguishable picture of the nanodimer modes coupling. ${ }^{45}$

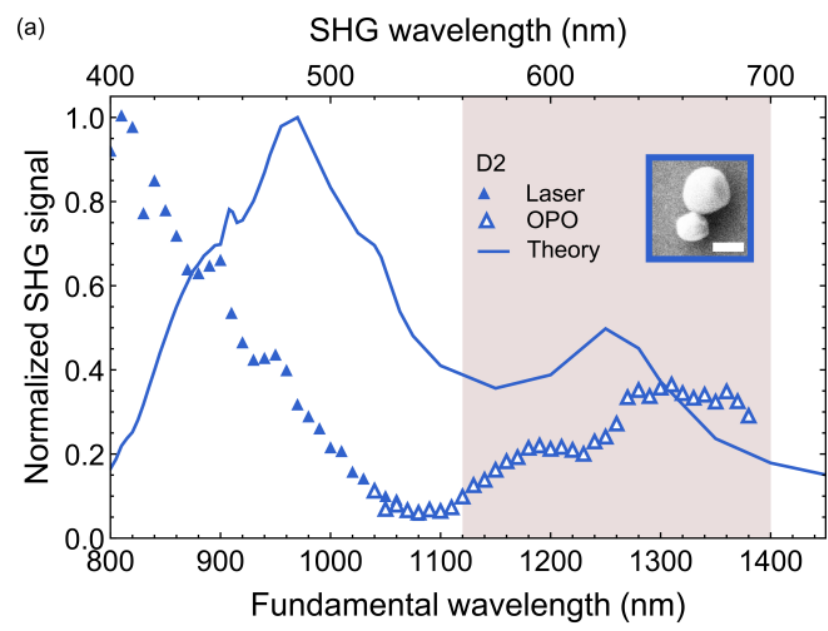

Polar dependence of SHG signal

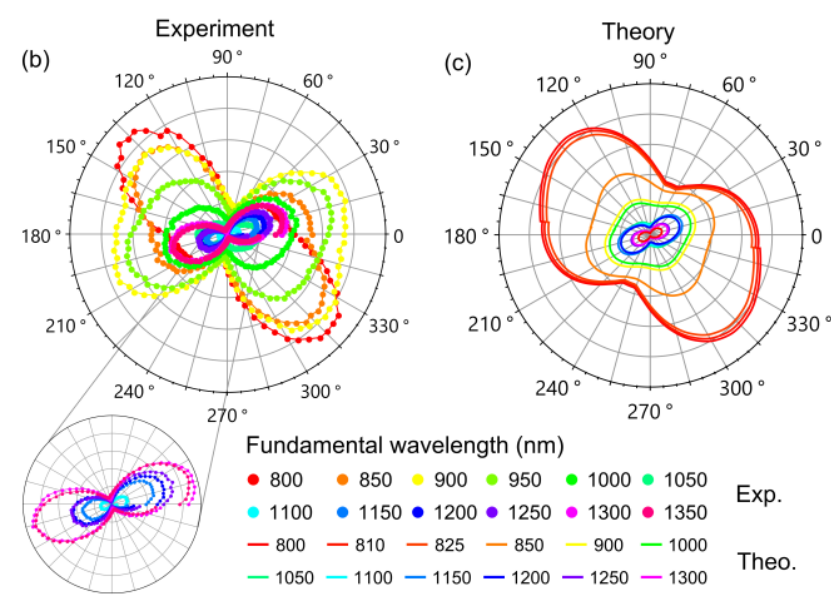

Figure 5. (a) Normalized SHG signal of nanodimer D2 as a function of the fundamental wavelength. The solid blue line shows the modeled spectrum and the blue triangles are the measurements points (filled laser, empty OPO). Micrograph of D2 in inset, scale bar $200 \mathrm{~nm}$. (b) Measured, (c) modeled polar dependence of the normalized SHG signal from nanodimer D2. The colors of the polar plots correspond to excitation wavelengths. 
We observe on the polarization dependent plots that the nanodimers undergo strong reshaping when the pumping wavelength changes (Figure $4 b-c$, Figure $5 b-c$, and Figure S12b-c in the supporting information). To explain the observed effect, we propose a basic theoretical model, which describes the SHG in a coupled dimer system. First, we neglect the SHG contribution from the gold nanoparticle as its efficiency is much lower than the BTO particle and we were not able to measure any signal coming from the single gold nanoparticle in the given experimental conditions. There are three main factors, which define the SHG response: i) the field distribution in the BTO nanoparticle at the fundamental wavelength, ii) the polar dependence of SHG in the BTO nanoparticle which is defined by the symmetry of modes and its crystalline structure; iii) the effect of the enhancement of the SHG emission due to the presence of the gold nanoparticle. Our simulations show that the third factor is less important for SHG than the resonant mode excitation at the fundamental wavelength, while the effect of the mode hybridization plays a crucial role in the reshaping of the polar dependence. Indeed, the mode hybridization leads to the redistribution of the electromagnetic energy stored in the BTO nanoparticle at the fundamental wavelength. In Figure 6a, numerical simulations obtained by direct integration of the electric energy over the BTO nanoparticle volume show the strong reshaping of the energy distribution inside the BTO nanoparticle. In the long wavelength range, the polar plots of energy in the BT nanoparticle are dictated by the presence of the gold particle and a hotspot formation, which results in strong SH response along the dimer axis. In the shorter wavelength region, the energy plots are also reshaped. This type of reshaping is discussed in detail in supporting information, section 2, within a basic dipole model. Next, in order to take into account the second factor related to the symmetry of $\chi^{(2)}$ tensor, ${ }^{44}$ we performed the simulations of SHG from a single BTO nanoparticle plotted in Figure 6b. Finally, the resulting polar dependence of the SHG in a nanodimer can be constructed by adding both contributions and multiplying the polar dependences shown in Figure 6a-b to obtain the polar dependences in Figure 6c, which fully describe the experimental results. The final SHG polar plots form nanodimers become strongly reshaped compared to a single BTO nanoparticle both in the short and long wavelength ranges. 

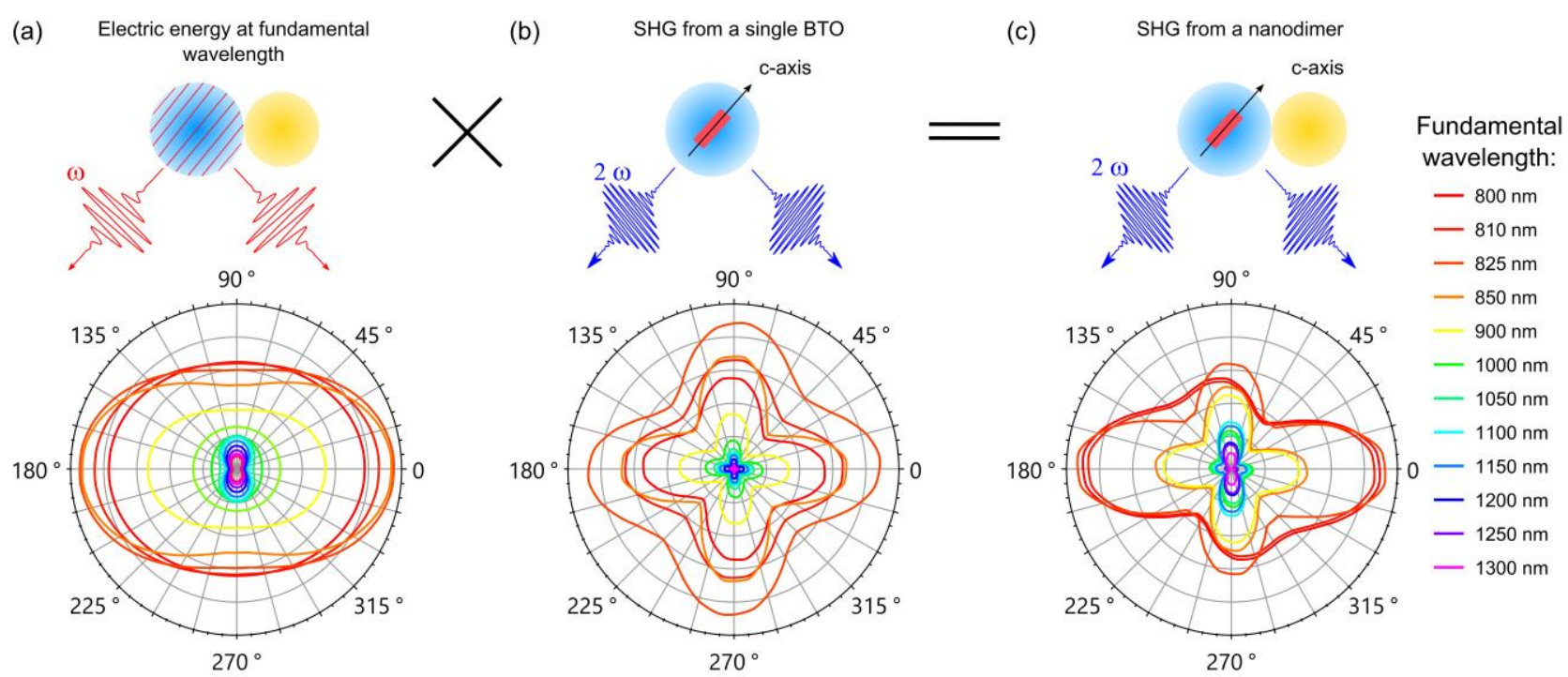

Figure 6. (a) Polarization dependence of the electric energy generated in the BTO nanoparticle at the fundamental wavelength. (b) Polarization dependence of the SHG signal of a single BTO nanoparticle. (c) Combined polar plots in (a) and (b) to get the SHG signal polar dependence of a hybrid nanodimer.

We then compare the SHG signal of five nanodimers, D1, D2 and three other nanodimers D3, D4 and D5 to the SHG signal of six different individual BTO nanoparticles. We evaluate the SHG enhancement of the nanodimers compared to BTO nanoparticles of the same size and in the same conditions. We select different BTO nanoparticles since the crystal structure orientation of the single BTO nanoparticle influence the SHG signal intensity. ${ }^{28}$ We find that for the laser region, we have enhancement from 2 to 22 and for the OPO region we have enhancement from 1 to 111 . The two orders of magnitude enhancement (111-fold) occurs in the OPO region where the hybridized mode is active. Moreover, we already compared the enhancement of a BTO nanoparticle of similar size to bulk BTO, and we obtained an enhancement of almost 5 orders of magnitude. ${ }^{35}$

In summary, we have reported experimental studies of nonlinear SHG response from hybrid nanodimers fabricated of Au and BTO nanoparticles by pick-and-place technique. With our specific design, we have achieved a strong hybridization of surface localized plasmon resonances and Mie resonances of metallic and dielectric nanoparticles combined into nanodimers. The hybridization is clearly revealed by direct measurements of the linear scattering spectra of individual nanoparticles and nanodimers in dark-field spectroscopy and by probing the hybridized resonance with the polarization dependency of the SHG signal in the nanodimers. We have confirmed the experimental data with numerical calculations of SHG from nanodimers. The hybrid nanodimers demonstrate strong reshaping 
of the polar plots from plain two-lobe shapes, provided by the field enhancement related to the plasmonic particle, to complex butterfly-type shapes, defined by the hybridized resonant modes and structure of the field inside the BTO nanoparticle. We have revealed the possibility to change the SHG polarization dependence through the wavelength and linear polarization state of the pump. We have reported also on the SHG signal enhancements linked to the hybridized mode with two orders of magnitude compared to single BTO nanoparticles. Reshaping of the second-harmonic polarization dependence demonstrated here can be used in a more general way in nonlinear optics to produce tunable nonlinear sources and to engineer light states through nonlinear near-field control. Hybrid systems have promising properties of tuning the directivity of the elastic scattering, so our results open a way for steering nonlinear emission. Indeed, the hybrid nanodimers could be used for optical excitation of nanoobjects in a controlled manner. From a broader point of view, the hybrid systems can act as a novel optical sensing platform combining functionalities of metallic and non-metallic materials in one. For instance, nanoscale sensing based on plasmon enhanced Raman scattering, luminescence enhancement of quantum emitters, or accessible thermal heating and temperature detection provided by non-metal systems can be envisioned. And now, with the reported results, we add controlled and efficient SHG light as a complementary optical characterization and detection tool widening the area of potential sensing applications of hybrid metaldielectric nanoantennas.

Methods. Far-field linear spectroscopy. Characterization of linear scattering cross sections were performed in a dark-field spectroscopy setup. We measure the linear scattering of single Au, BTO nanoparticle and hybrid nanodimers. We show in Fig. S1 of the supporting information the dark-field measurement setups for the linear backward and forward scattering measurements. ${ }^{28,46}$ The single nanoobjects (single $\mathrm{Au}, \mathrm{BTO}$ or nanodimer) are illuminated by a halogen lamp through a $50 \times(\mathrm{NA}=$ 0.55) dark-field objective for the reflection measurements. In the case of transmission measurements, they are illuminated from the back side of the substrate with a dark-field condenser. The scattering of each nanoobjects is collected with the same objective and measured with an imaging spectrometer through a multimode fiber. The fiber acts as a pinhole and collects only the signal around an individual object. The diameter of the collection area in this setup configuration is $5 \mu \mathrm{m}$. The nanoobject is 
positioned within the collection area of the optical fiber. The measured signal is corrected by the background contributions and the wavelength dependence of the halogen lamp spectrum.

Nonlinear optical characterization. Nonlinear optical measurements of the SHG spectra were performed with a home-built transmission optical setup (Supporting Information). For the excitation of the samples, we used a tunable Ti:Sapphire laser together with an optical parametric oscillator (with an average pulse duration of $200 \mathrm{fs}$ over the $690-1600 \mathrm{~nm}$ range and a $80 \mathrm{MHz}$ repetition rate) in the wavelength range from $800-1400 \mathrm{~nm}$, with steps of $10 \mathrm{~nm}$. The laser light was focused with a $11 \mathrm{~mm}$ effective focal aspheric lens on the nanostructure through the backside of the ITO covered glass with isolated hybrid nanodimers and single BTO nanoparticles. The signal was then collected with a 100x objective (NA= 0.8 ) and focused onto a scientific CMOS camera. The polarization of the excitation laser was fixed for all steps of the spectral measurements. For the polar dependence of the SHG of the hybrid nanodimers, the excitation polarization is rotated of $180^{\circ}$ by $2^{\circ}$ steps (corresponding to a full circle). As a reference sample for the determination of the enhancement factor, we measured the SHG spectra from various single BTO nanoparticles with a diameter close to the ones used in the hybrid nanodimers. The corresponding SHG spectra from the single BTO nanoparticles and the hybrid nanodimers were normalized by the transmission of the optics and filters as well as the laser power level, the integration time of the camera, its quantum efficiency, the pulse duration of the optical setup (measured for all the wavelength at the sample position), the beam size of the fundamental and the one of the SHG signal (Supporting Information). We also measured the dependence of the SHG signal intensity for different incident power and observed a typical quadratic response.

Numerical FEM simulations. The numerical simulations were performed using the COMSOL Multiphysics software package. For the numerical simulations of the scattering cross section of the hybrid nanodimers structures and the SHG spectra and polar plots for the different geometries we developed a model. In our numerical model we are calculating how much is collected in the far-field by considering the numerical aperture (NA) of the collection objective.

\section{ASSOSIATED CONTENT}




\section{Supporting Information}

More details about experimental methods, fabrication and theoretical analysis are presented in Supporting Information.

This material is available free of charge via Internet at http://pubs.acs.org.

\section{AUTHOR INFORMATION}

\section{Corresponding Authors}

*E-mail: grange@phys.ethz.ch

\section{Author Contributions}

The manuscript was written through contributions of all authors. All authors have given approval to the final version of the manuscript.

C.R. performed the linear optical characterization. C.R. and L.L. performed the second-harmonic generation measurements and data analysis with the help of M.T. K.F. and M.P. did the theoretical modelling. F.T. helped with the linear and nonlinear measurements. F.E.K. and I.S.M. fabricated the dimers by pick-and-place methods. Y.K. and R.G. supervised the project.

\section{ACKNOWLEDGMENTS}

The authors thank the Scientific Centre for Optical and Electron Microscopy (ScopeM) of ETH Zurich and acknowledge the financial supports from the Swiss National Science Foundation (SNF) (163916 and 150609), and the ERC starting grant Chi2-Nano-Oxide (714837). The dimers samples were fabricated under the support of Russian Science Foundation (RSF) Project No RFME-FI58416X0018. We would like to thank Teresa and Vincenzo Buscaglia (CNR, Genova, Italy) for synthesizing and providing the $\mathrm{BaTiO}_{3}$ nanoparticles.

\section{REFERENCES}

(1) Schuller, J. A.; Barnard, E. S.; Cai, W.; Jun, Y. C.; White, J. S.; Brongersma, M. L. Plasmonics for Extreme Light Concentration and Manipulation. Nat. Mater. 2010, 9 (3), 193-204. 
(2) Bonod, N.; Devilez, A.; Rolly, B.; Bidault, S.; Stout, B. Ultracompact and Unidirectional Metallic Antennas. Phys. Rev. B - Condens. Matter Mater. Phys. 2010, 82 (11), 115429.

(3) Hancu, I. M.; Curto, A. G.; Castro-López, M.; Kuttge, M.; van Hulst, N. F. Multipolar Interference for Directed Light Emission. Nano Lett. 2014, 14 (1), 166-171.

(4) Butet, J.; Bernasconi, G. D.; Petit, M.; Bouhelier, A.; Yan, C.; Martin, O. J. F.; Cluzel, B.; Demichel, O. Revealing a Mode Interplay That Controls Second-Harmonic Radiation in Gold Nanoantennas. ACS Photonics 2017, 4 (11), 2923-2929.

(5) Boyd, R. W. Nonlinear Optics; Academic Press, 2008.

(6) Kauranen, M.; Zayats, A. V. Nonlinear Plasmonics. Nat. Photonics 2012, 6 (11), 737-748.

(7) Butet, J.; Brevet, P.-F.; Martin, O. J. F. Optical Second Harmonic Generation in Plasmonic Nanostructures: From Fundamental Principles to Advanced Applications. ACS Nano 2015, 9 (11), $10545-10562$.

(8) Panoiu, N. C.; Sha, W. E. I.; Lei, D. Y.; Li, G.-C. Nonlinear Optics in Plasmonic Nanostructures. J. Opt. 2018, 20 (8), 083001.

(9) Krasavin, A. V.; Ginzburg, P.; Zayats, A. V. Free-Electron Optical Nonlinearities in Plasmonic Nanostructures: A Review of the Hydrodynamic Description. Laser Photonics Rev. 2018, 12 (1), $1-24$.

(10) Kuznetsov, A. I.; Miroshnichenko, A. E.; Brongersma, M. L.; Kivshar, Y. S.; Luk’yanchuk, B. Optically Resonant Dielectric Nanostructures. Science. 2016, 354 (6314).

(11) Kruk, S.; Kivshar, Y. Functional Meta-Optics and Nanophotonics Governed by Mie Resonances. ACS Photonics 2017, 4 (11), 2638-2649.

(12) Vahala, K. J. Optical Microcavities. Nature 2003, 424 (6950), 839-846.

(13) Yavas, O.; Svedendahl, M.; Dobosz, P.; Sanz, V.; Quidant, R. On-a-Chip Biosensing Based on 
All-Dielectric Nanoresonators. Nano Lett. 2017, 17 (7), 4421-4426.

(14) Tittl, A.; Leitis, A.; Liu, M.; Yesilkoy, F.; Choi, D.-Y.; Neshev, D. N.; Kivshar, Y. S.; Altug, H. Imaging-Based Molecular Barcoding with Pixelated Dielectric Metasurfaces. Science (80-. ). 2018, 360 (6393), 1105 LP-1109.

(15) Liu, S.; Sinclair, M. B.; Saravi, S.; Keeler, G. A.; Yang, Y.; Reno, J.; Peake, G. M.; Setzpfandt, F.; Staude, I.; Pertsch, T.; et al. Resonantly Enhanced Second-Harmonic Generation Using III-V Semiconductor All-Dielectric Metasurfaces. Nano Lett. 2016, 16 (9), 5426-5432.

(16) Smirnova, D.; Kivshar, Y. S. Multipolar Nonlinear Nanophotonics. Optica 2016, 3 (11), 1241.

(17) Gili, V. F.; Carletti, L.; Locatelli, A.; Rocco, D.; Finazzi, M.; Ghirardini, L.; Favero, I.; Gomez, C.; Lemaître, A.; Celebrano, M.; et al. Monolithic AlGaAs Second-Harmonic Nanoantennas. Opt. Express 2016, 24 (14), 6488-6492.

(18) Camacho-Morales, R.; Rahmani, M.; Kruk, S.; Wang, L.; Xu, L.; Smirnova, D. A.; Solntsev, A. S.; Miroshnichenko, A.; Tan, H. H.; Karouta, F.; et al. Nonlinear Generation of Vector Beams from AlGaAs Nanoantennas. Nano Lett. 2016, 16 (11), 7191-7197.

(19) Timofeeva, M.; Lang, L.; Timpu, F.; Renaut, C.; Bouravleuv, A.; Shtrom, I.; Cirlin, G.; Grange, R. Anapoles in Free-Standing III-V Nanodisks Enhancing Second-Harmonic Generation. Nano Lett. 2018, 18 (6), 3695-3702.

(20) Makarov, S. V.; Petrov, M. I.; Zywietz, U.; Milichko, V.; Zuev, D.; Lopanitsyna, N.; Kuksin, A.; Mukhin, I.; Zograf, G.; Ubyivovk, E.; et al. Efficient Second-Harmonic Generation in Nanocrystalline Silicon Nanoparticles. Nano Lett. 2017, 17 (5), 3047-3053.

(21) Carletti, L.; Koshelev, K.; De Angelis, C.; Kivshar, Y. Giant Nonlinear Response at the Nanoscale Driven by Bound States in the Continuum. Phys. Rev. Lett. 2018, 121 (3), 033903.

(22) Lepeshov, S.; Krasnok, A. E.; Belov, P. A.; Miroshnichenko, A. E. Hybrid Nanophotonics. Phys. Usp. 2017. 
(23) Decker, M.; Pertsch, T.; Staude, I. Strong Coupling in Hybrid Metal-Dielectric Nanoresonators. Philos. Trans. A. Math. Phys. Eng. Sci. 2017, 375 (2090), 20160312.

(24) Novotny, L.; van Hulst, N. Antennas for Light. Nat. Photonics 2011, 5 (2), 83-90.

(25) CF Bohren, D. H. Absorption and Scattering of Light by Small Particles.; John Wiley \& Sons.: New York, NY, 2008.

(26) Metzger, B.; Hentschel, M.; Schumacher, T.; Lippitz, M.; Ye, X.; Murray, C. B.; Knabe, B.; Buse, K.; Giessen, H. Doubling the Efficiency of Third Harmonic Generation by Positioning ITO Nanocrystals into the Hot-Spot of Plasmonic Gap-Antennas. Nano Lett. 2014, 14 (5), 2867-2872.

(27) Linnenbank, H.; Grynko, Y.; Förstner, J.; Linden, S. Second Harmonic Generation Spectroscopy on Hybrid Plasmonic/Dielectric Nanoantennas. Light Sci. Appl. 2016, 5 (1), e16013.

(28) Timpu, F.; Hendricks, N. R.; Petrov, M.; Ni, S.; Renaut, C.; Wolf, H.; Isa, L.; Kivshar, Y.; Grange, R. Enhanced Second-Harmonic Generation from Sequential Capillarity-Assisted Particle Assembly of Hybrid Nanodimers. Nano Lett. 2017, 17 (9), 5381-5388.

(29) Liu, W.; Kivshar, Y. S. Multipolar Interference Effects in Nanophotonics. Philos. Trans. A. Math. Phys. Eng. Sci. 2017, 375 (2090), 20160317.

(30) Wang, H.; Liu, P.; Ke, Y.; Su, Y.; Zhang, L.; Xu, N.; Deng, S.; Chen, H. Janus Magneto-Electric Nanosphere Dimers Exhibiting Unidirectional Visible Light Scattering and Strong Electromagnetic Field Enhancement. ACS Nano 2015, 9 (1), 436-448.

(31) Sun, S.; Li, M.; Du, Q.; Png, C. E.; Bai, P. Metal-Dielectric Hybrid Dimer Nanoantenna: Coupling between Surface Plasmons and Dielectric Resonances for Fluorescence Enhancement. J. Phys. Chem. C 2017, 121 (23), 12871-12884.

(32) Rusak, E.; Staude, I.; Decker, M.; Sautter, J.; Miroshnichenko, A. E.; Powell, D. A.; Neshev, D. N.; Kivshar, Y. S. Hybrid Nanoantennas for Directional Emission Enhancement. Appl. Phys. Lett. 2014, 105 (22), 221109. 
(33) Zuev, D. A.; Makarov, S. V.; Mukhin, I. S.; Milichko, V. A.; Starikov, S. V.; Morozov, I. A.; Shishkin, I. I.; Krasnok, A. E.; Belov, P. A. Fabrication of Hybrid Nanostructures via Nanoscale Laser-Induced Reshaping for Advanced Light Manipulation. Adv. Mater. 2016, 28 (16), 30873093.

(34) Markovich, D.; Baryshnikova, K.; Shalin, A.; Samusev, A.; Krasnok, A.; Belov, P.; Ginzburg, P. Enhancement of Artificial Magnetism via Resonant Bianisotropy. Sci. Rep. 2016, 6 (1), 22546.

(35) Timpu, F.; Sergeyev, A.; Hendricks, N. R.; Grange, R. Second-Harmonic Enhancement with Mie Resonances in Perovskite Nanoparticles. ACS Photonics 2017, 4 (1), 76-84.

(36) Denisyuk, A. I.; Komissarenko, F. E.; Mukhin, I. S. Electrostatic Pick-and-Place Micro/Nanomanipulation under the Electron Beam. Microelectron. Eng. 2014, 121, 15-18.

(37) Denisyuk, A. I.; Krasavin, A. V.; Komissarenko, F. E.; Mukhin, I. S. Mechanical, Electrostatic, and Electromagnetic Manipulation of Microobjects and Nanoobjects in Electron Microscopes. Adv. Imaging Electron Phys. 2014, 186, 101-140.

(38) Mock, J. J.; Smith, D. R.; Schultz, S. Local Refractive Index Dependence of Plasmon Resonance Spectra from Individual Nanoparticles. Nano Lett. 2003, 3 (4), 485-491.

(39) Su, K. H.; Wei, Q. H.; Zhang, X.; Mock, J. J.; Smith, D. R.; Schultz, S. Interparticle Coupling Effects on Plasmon Resonances of Nanogold Particles. Nano Lett. 2003, 3 (8), 1087-1090.

(40) Timofeeva, M.; Bouravleuv, A.; Cirlin, G.; Shtrom, I.; Soshnikov, I.; Reig Escalé, M.; Sergeyev, A.; Grange, R. Polar Second-Harmonic Imaging to Resolve Pure and Mixed Crystal Phases along GaAs Nanowires. Nano Lett. 2016, 16 (10), 6290-6297.

(41) Chervinskii, S.; Koskinen, K.; Scherbak, S.; Kauranen, M.; Lipovskii, A. Nonresonant Local Fields Enhance Second-Harmonic Generation from Metal Nanoislands with Dielectric Cover. Phys. Rev. Lett. 2018, 120 (11), 113902.

(42) Kim, E.; Steinbrück, A.; Buscaglia, M. T.; Buscaglia, V.; Pertsch, T.; Grange, R. Second- 


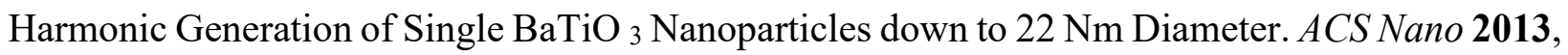
$7(6), 5343-5349$.

(43) Hsieh, C.-L.; Pu, Y.; Grange, R.; Psaltis, D. Second Harmonic Generation from Nanocrystals under Linearly and Circularly Polarized Excitations. Opt. Express 2010, 18 (11), 11917.

(44) Frizyuk, K.; Volkovskaya, I.; Smirnova, D.; Poddubny, A.; Petrov, M. Second-Harmonic Generation in Mie-Resonant Dielectric Nanoparticles Made of Noncentrosymmetric Materials. arXiv Opt. 2018, 1809.06456.

(45) Bouhelier, A.; Beversluis, M.; Hartschuh, A.; Novotny, L. Near-Field Second-Harmonic Generation Induced by Local Field Enhancement. Phys. Rev. Lett. 2003, 90 (1), 13903.

(46) Timpu, F.; Sergeyev, A.; Hendricks, N. R.; Grange, R. Second-Harmonic Enhancement with Mie Resonances in Perovskite Nanoparticles. ACS Photonics 2017, 4 (1), 76-84. 